\title{
A single center analysis of factors influencing study start-up timeline in clinical trials
}

\begin{abstract}
Aim: Efficient start-up phase in clinical trials is crucial to execution. The goal was to determine factors contributing to delays. Materials \& methods: The start-up milestones were assessed for 38 studies and analyzed. Results: Total start-up time was shorter for following studies: device trials, no outsourcing, fewer ancillary services used and in interventional versus observational designs. The use of a centralized Institutional Review Board (IRB) versus a local IRB reduced time to approval. Studies that never enrolled took longer on average to finalize their budget/contract, and obtain IRB than ones that did enroll. Conclusion: Different features of clinical trials can affect timeline of start-up process. An understanding of the impact of each feature allows for optimization.
\end{abstract}

Lay Abstract: Prompt start-up phase in clinical trials is critical to successful execution. The goal of this manuscript was to determine factors contributing to delays. We assessed factors affecting start-up for 38 studies. Total start-up time was shorter for studies of device trials, without outsourcing, using fewer ancillary services and with certain designs. The use of a centralized Institutional Review Board (IRB) versus a local IRB reduced time to approval. Studies that never enrolled took longer on average to finalize their budget/contract, and obtain IRB than ones that did enroll. We conclude that different factors can affect timeline of start-up process. An understanding of the impact of each factor allows for optimization.

First draft submitted: 21 February 2017; Accepted for publication: 19 May 2017; Published online: 14 July 2017

Keywords: clinical trials management $\bullet$ process optimization $\bullet$ start-up metrics

- start-up phase $\bullet$ trial efficiency

The study start-up phase at the site level represents a significant administrative and logistical undertaking. The initiation of a new clinical trial typically involves completion of a feasibility questionnaire, budget and contract negotiation, Institutional Review Board (IRB) preparation and submission, regulatory essential documents collection, establishment of study logistics in collaboration with ancillary services within the institution and development a patient recruitment strategy [1]. Successful navigation of this phase in any clinical trial life cycle is critical to the successful initiation of a study in a timely manner. Nevertheless, the different stages of start-up phase vary in duration among sites, and there are few standardized measurements of milestones, making it difficult to know how well a site is doing relative to others [2].

Based on our experience, we feel that complex study protocols and procedures that involve an increased number of ancillary services (i.e., services provided by different departments and/or external services within
Brianna M Krafcik', Gheorghe Doros² \& Marina A Malikova*,1

'Boston University, Boston Medical Center, Department of Surgery, Boston, MA, USA

${ }^{2}$ Boston University, Department of Biostatistics, Boston, MA, USA ${ }^{*}$ Author for correspondence: mmalikov@bu.edu 
the same institution such as Blood Bank, Investigational Drug Services pharmacy, imaging core facilities, pathology laboratory facilities, etc.), requirement for Institutional Biosafety Committee (IBC) approval and inclusion of interventional procedures, can increase the expected amount of time required to complete the study start-up process. The IBC Institutional Biosafety is an institutional committee created under the NIH Guidelines to review research involving recombinant DNA and synthetic nucleic acid research. The IBC also reviews all research involving biohazardous materials (i.e., biological specimens collected during the course of the study such as blood, urine, tissue samples, etc.) and has overall oversight and responsibility for the biosafety program at the organization (i.e., hospital, university, private practice). The IBC carries out these functions pursuant to requirements set forth by federal, state and local agencies, as well as organization.

Additionally, extrinsic features of studies such as Contract Research Organization (CRO) utilization, project manager experience and the use of a local versus centralized IRB tend to impact the amount of time the study start-up will take [1].

The goal of the current investigation was to identify each factor that may play a role in delaying or expediting the start-up phase of a clinical study to define what is most important in determining the time from initial contact of a potential clinical site by sponsor or its representatives (i.e., CRO) to first patient enrollment. We used the data available at our site from previous study start-ups to characterize the role each element of a study has in regards to the time to reach milestones during the initiation process. Given the unique features of each individual study, it is difficult to use universal benchmarks for study start-up performance and apply them to all studies [2]. However, stratification by study type and characteristics can allow for a better understanding of realistic timelines for future studies sharing the same features.

\section{Materials \& methods}

Thirty-eight industry-sponsored clinical studies conducted at Boston University/Boston Medical Center (BU/BMC) in the Department of Surgery between 2004 and 2016 were analyzed to determine the datespecific start-up metrics were achieved. These metrics included date of initial contact (date of feasibility questionnaire completion), date contract and budget were received at the site, date the contract and budget were submitted to the appropriate institutional administrators and number of days between contract and budget receipt from the sponsor and finalization. Additionally, date of site receipt of the protocol and informed consent form (ICF) from the sponsor, date of submission to the IRB and date of IRB approval were recorded. Number of days to IRB approval was calculated for the time between protocol/ICF receipt from the sponsor and IRB approval as well as the time between protocol submission to the IRB and IRB approval. Specifically, the number of days from receipt from the Sponsor to

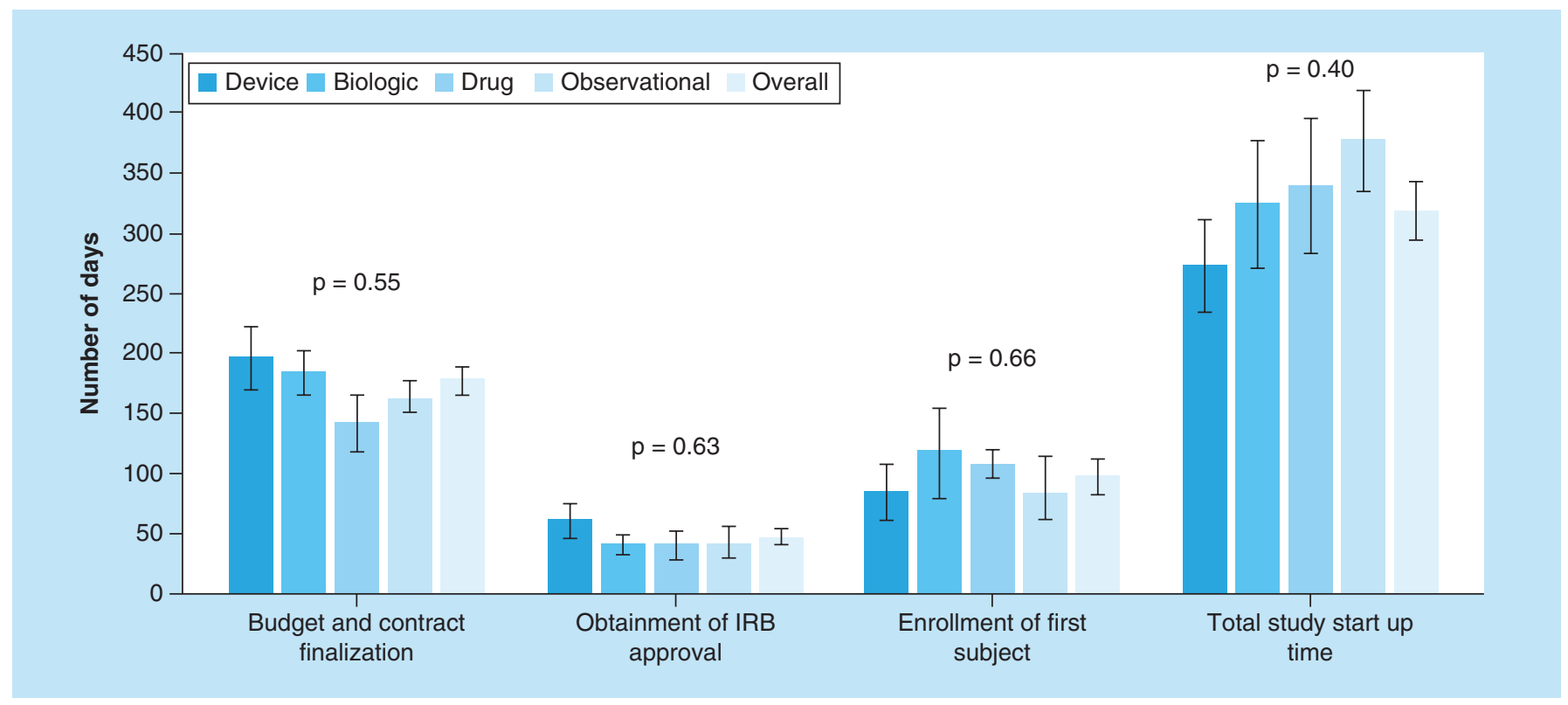

Figure 1. Comparison of number of days to finalize budget and contract, days to Institutional Review Board approval, days to first subject enrollment and total start-up time between different study types. Total study start-up time is defined as site receipt of the feasibility questionnaire to first subject enrollment. Bars represent standard error for each mean value. IRB: Institutional Review Board. 
Table 1. Median and 75th percentile number of days to budget and contract finalization, Institutional Review Board approval and first subject enrollment for each study type.

\begin{tabular}{|llll|}
\hline & Budget/contract negotiation & IRB approval & First subject enrolled \\
\hline Drug trials & & & \\
\hline 75th percentile & 170 & 72.25 & 127 \\
\hline 50th percentile & 131 & 37 & 109 \\
\hline Biologic trials & & 56 & 198.75 \\
\hline 75th percentile & 234 & 39 & 87.5 \\
\hline 50th percentile & 178 & & 134.5 \\
\hline Device trials & & 103 & 68.5 \\
\hline 75th percentile & 274 & 61 & 149.75 \\
\hline 50th percentile & 168 & & 54 \\
\hline Observational trials & & 69.5 & \\
\hline 75th percentile & 188 & 30 & \\
\hline 50th percentile & 163 & & \\
\hline IRB: Institutional Review Board. & & & \\
\hline
\end{tabular}

IRB submission is relevant since its measures how long the initial regulatory submission package was in the hands of the site research team in preparation for IRB submission. This is versus time from IRB submission to IRB approval, which defines how it took for the IRB to review and approve the study.

The date of first subject enrollment was collected and used to determine the number of days from IRB approval to patient enrollment as well as the total number of start-up days, from feasibility questionnaire completion to first subject enrollment. Additional study features such as local versus centralized IRB utilization, outsourcing of services to a CRO by project Sponsor, requirement of IBC approval, clinical research site project manager's experience and number of ancillary services was also collected. Dates and study information was collected from the corresponding regulatory binder and was supplemented with previous correspondence between our site and the sponsors, CROs and institutional personnel.

Statistical analysis was performed on the number of days to achieve each metric, specifically days to contract and budget finalization, IRB approval, first subject enrollment and total study start-up time. The mean number of days and standard error for each milestone was considered in the context of type of study (drug as traditional single molecule chemical entity produced via organic synthesis; device - medical machine/apparatus, contrivance, implant, in vitro reagent; biologic as macromolecular produced via recombinant DNA technology or isolated from biological organism - that is, vaccine, gene therapy, proteins, cytokines, tissue transplants; or observational), presence or absence of a CRO, project manager experience (more or less than 48 months' experience for all studies), number of ancillary services ( 0 -none, between one or two: $1-2$, between three to four: $3-4$, more than five: $5+$ ), local versus centralized IRB and interventional versus observational studies. The $t$-test and analysis of variance were used to compare average number of days within each group to determine statistical significance with $\mathrm{p}=<0.05$ defined as statistically significant. Additionally, the 50th and 75th percentiles were determined for number of days to finalize contract and budget and to obtain IRB approval for each study type in order to establish a benchmark of comparison for number of days to achieve each milestone. While collecting the data, reasons for study delay or termination were noted and incorporated into a cause-effect analysis. Multivariate analysis was then conducted to assess each milestone as a combined function of IRB type, use of a CRO and project manager experience, as these were considered to be the most important factors influencing study start-up based on the t-test and analysis of variance.

Additionally, in an effort to determine which factors may cause a study not to progress past a specific stage in the start-up process, analysis was conducted on the percentage of studies that failed in each stage of the start-up process stratified by relevant characteristics. Based on this concept, we also completed a KaplanMeier-type analysis to depict whether an extended number of days to finalize contract and budget or to obtain IRB approval caused the study to fail in the following stages. For example, we sought to determine whether a long amount of time to obtain IRB 
approval was associated with a decreased likelihood to ever enroll a first patient. The formula $S\left(t_{x+1}\right)=S\left(t_{x}\right)$ $\left(1-d_{x} / n_{x}\right)$ was used, where $t_{x}$ is the number of days, $d_{x}$ is the number of studies terminated on that day, $\mathrm{n}_{\mathrm{x}}$ is the number of studies active on that day, respectively, and S represents the probability of 'success' or continuation to the next start-up milestone. Days to contract and budget approval was marked on the $\mathrm{x}$ axis of the first analysis, and probability of obtaining IRB approval and enrolling a first patient was plotted as a function of the number of elapsed days. Similarly, days to IRB approval were indicated on the $\mathrm{x}$ axis of a separate analysis and probability of enrolling a patient was portrayed.

\section{Results}

The 50th and 75th percentile of the number of days to finalize the contract and budget, obtain IRB approval and enroll a subject stratified by study type is presented in Table 1. A comparison of the average number of days to reach each of the three milestones as well as total study start-up time by study type is shown in Figure 1 . When comparing average number of days to reach each milestone between study types, drug studies took the least time to achieve a fully negotiated contract and budget (141.4 days), while device studies took the longest (196.3 days). The average number of days for all studies considered together to finalize the budget and contract was 177.6 days. The difference between study types was not statistically significant $(\mathrm{p}=0.55)$. The number of days to obtain IRB approval once submitted was similar between study types, however, was the least in biologic studies (39.8 days) and the longest in device studies (59.7 days). This again was not statistically significant ( $p=0.63$ ), and overall number of days on average to approval was 46.8 days. Time to enroll the first subject following IRB approval was the least in observational studies at 82 days and the most in biologic studies at 116.7 days $(\mathrm{p}=0.66)$. Total study start-up time from initial site contact to first patient enrollment was the least in device studies (274 days) and the most in observational studies (377.5 days), with an overall average of 318.8 days, and the difference between study types was not statistically significant $(\mathrm{p}=0.40)$.

As depicted in Figure 2, the use of a CRO decreased the number of days to contract and budget finalization (171.8 days with a CRO vs 184.9 days without, $\mathrm{p}=0.60)$ and days to obtain IRB approval (41.8 days vs 52.6 days, $\mathrm{p}=0.41)$. Time to first patient enrollment following IRB approval (108.5 days vs 80.6 days, $\mathrm{p}=0.37$ ) and total amount of time for study start-up (335 days vs 295.4 days, $\mathrm{p}=0.34$ ) were increased with a CRO. None of these differences was statistically significant.

When considering the impact of project manager experience, more experience reduced the number of days for the contract and budget finalization process from 192.9 to 162.3 days $(\mathrm{p}=0.58)$. More experience

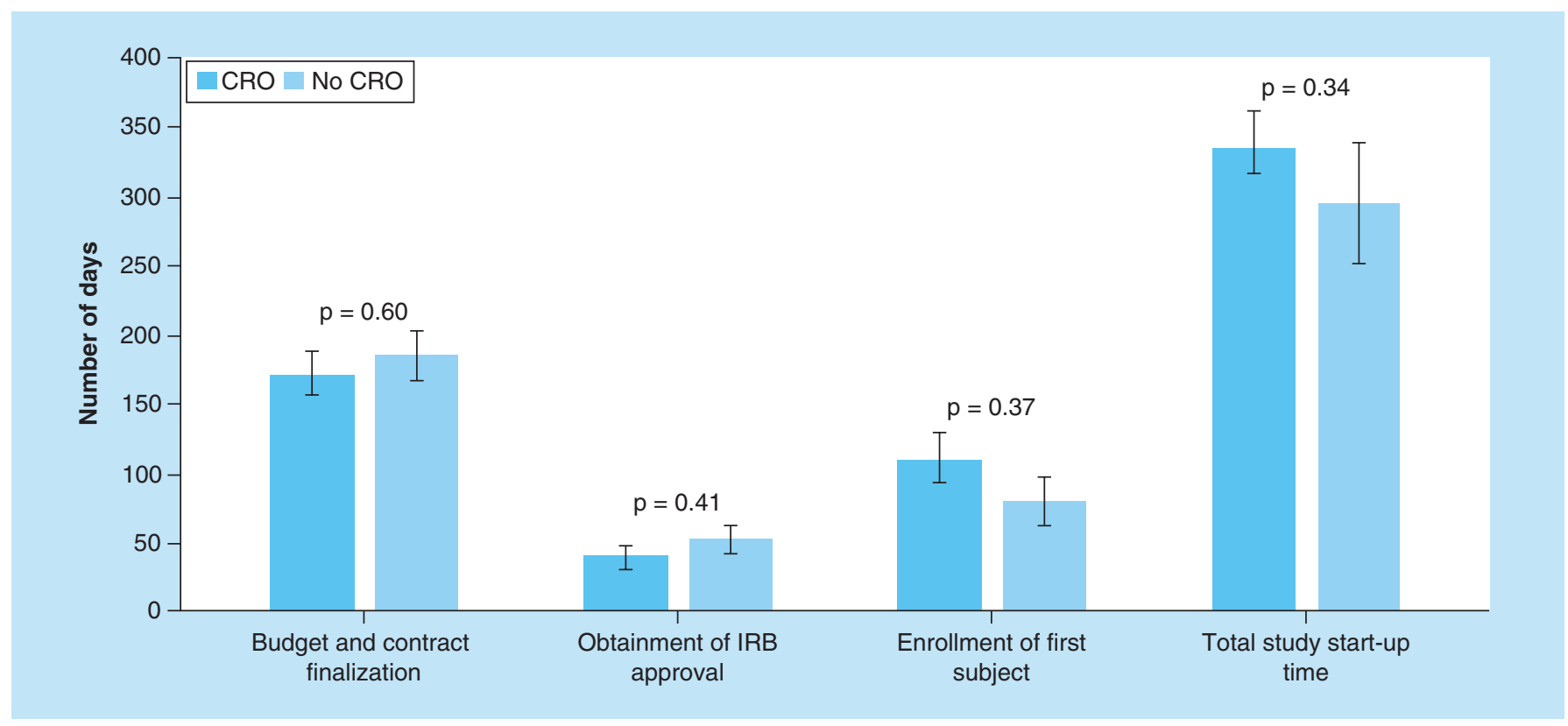

Figure 2. Comparison of number of days to finalize budget and contract, days to Institutional Review Board approval, days to first subject enrollment and total study start-up time with the presence and absence of a Contract Research Organization working on the study. Total study start-up is defined as date of site receipt of the feasibility questionnaire to first subject enrollment. Bars represent standard error for each mean value.

CRO: Contract Research Organization; IRB: Institutional Review Board. 


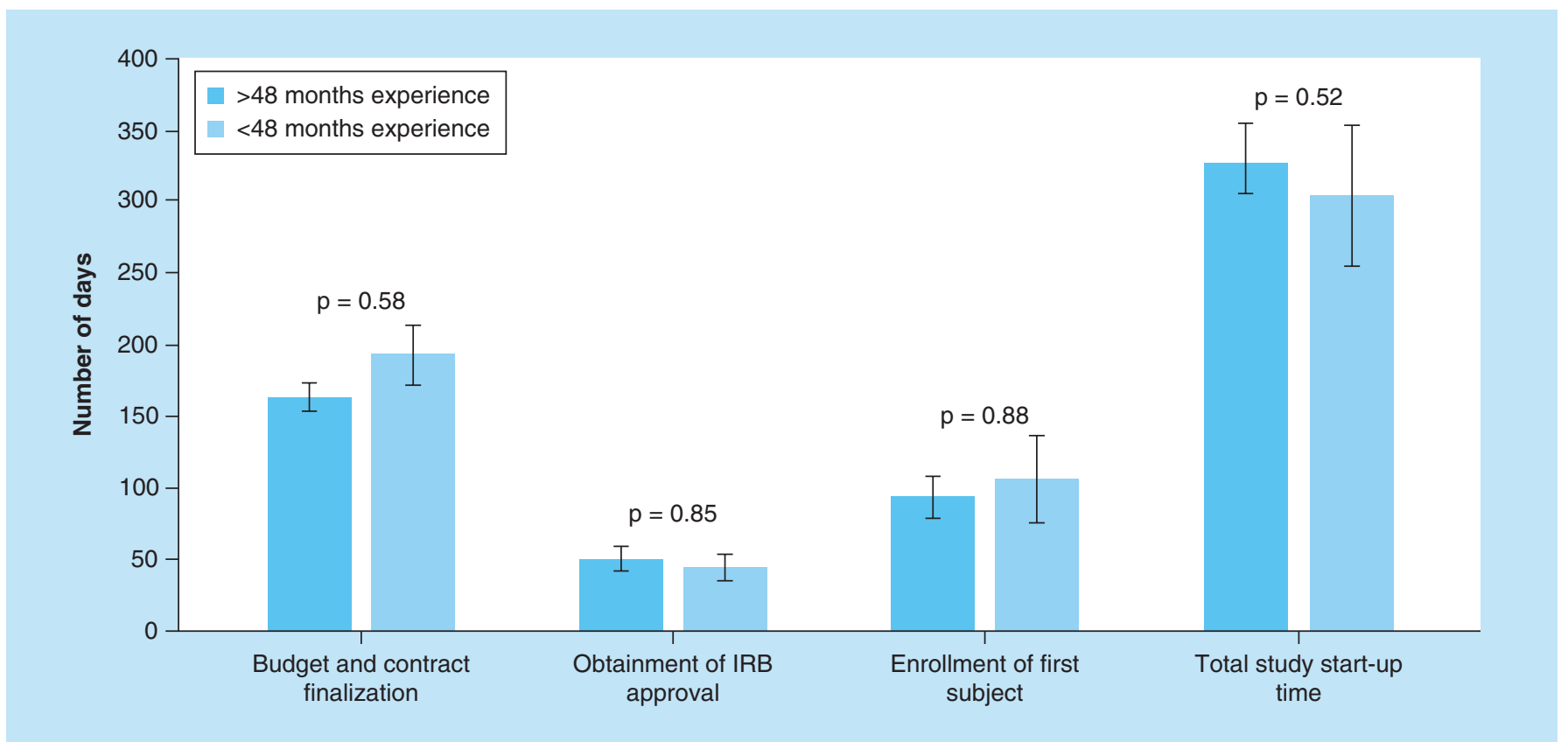

Figure 3. Comparison of number of days to finalize budget and contract, days to Institutional Review Board approval, days to first subject enrollment and total study start-up time based on project manager experience. Total study start-up is defined as date of site receipt of the feasibility questionnaire to first subject enrollment. Forty-eight months was the approximate median experience time for all studies considered. Bars represent standard error for each mean value.

IRB: Institutional Review Board.

also reduced the time between IRB approval and first patient enrollment from 106.1 days to 91.9 days $(\mathrm{p}=$ 0.88). Time to IRB approval was slightly increased with project manager experience greater than 48 months, but only by 4.6 days - from 44.4 to 49 days on average $(\mathrm{p}=0.85)$. The total start-up time was somewhat longer with a more experienced project manager as compared with a less experienced manager (327.4 vs 303.9 days, $\mathrm{p}=0.52)$. These results are depicted in Figure 3 , and none of the results were statistically significant.

The effect of ancillary services on study start-up time is shown in Figure 4. An increased number of ancillary services required by the study protocol was correlated with an increase in the average amount of time to finalize the contract and budget $(0$ services $=117$ days, $1-2$ services $=172$ days, $3-4$ services $=193.5$ days, $5+$ services $=163$ days, $\mathrm{p}=0.60)$, time to enroll the first subject $(0=79.5$ days, $1-2=83.8$ days, $3-4=99$ days, $5+=158.5$ days, $\mathrm{p}=0.74)$ and total start-up time $(0$ $=259$ days, $1-2=318.1$ days, $3-4=337.3$ days, $5+=$ 289 days, $\mathrm{p}=0.89$ ), particularly when three or more services were involved. Days to IRB approval showed a similar trend, although studies with no services took longer to be approved than studies with between 1 and 3 services $(0=58$ days, $1-2=54.8$ days, $3-4=$ 35.9 days, $5+=55.5$ days, $\mathrm{p}=0.33$ ).

The requirement of IBC approval for studies in conjunction with IRB approval reduced the time between IRB submission and approval from 47.4 days to 45.3 days on average, as shown in Figure 5 , although the difference was not statistically significant $(\mathrm{p}=$ 0.55 ). As seen in Figure 6 , use of a centralized IRB did decrease the time between IRB submission and approval as compared with use of a local IRB, from 65.9 days to 30.1 days. This difference approached statistical significance $(\mathrm{p}=0.07)$.

In comparing interventional (drug, device or biologic) studies with observational studies, observational studies had faster budget and contract finalization (156 days vs 179.6 days, $\mathrm{p}=0.86$ ), IRB approval (41 days vs 47.1 days, $\mathrm{p}=0.84$ ) and first subject enrollment (82 days vs 100.4 days, $\mathrm{p}=0.71$ ). Total study start-up time, however, was less for interventional as compared with observational studies (305.7 vs 377.8 days, $\mathrm{p}=0.12$ ). These results are shown in Figure 7, and none of the differences were statistically significant.

When comparing the amount of time to finalize the contract and budget for studies that eventually obtained IRB approval as compared with those that ultimately did not, studies that never obtained IRB approval had a longer budget and contract finalization process versus those that did obtain IRB approval (211.8 vs 173.3 days, $\mathrm{p}=0.81$ ). Similarly, the budget and contract finalization process took longer in studies which ultimately did not enroll a patient as compared 


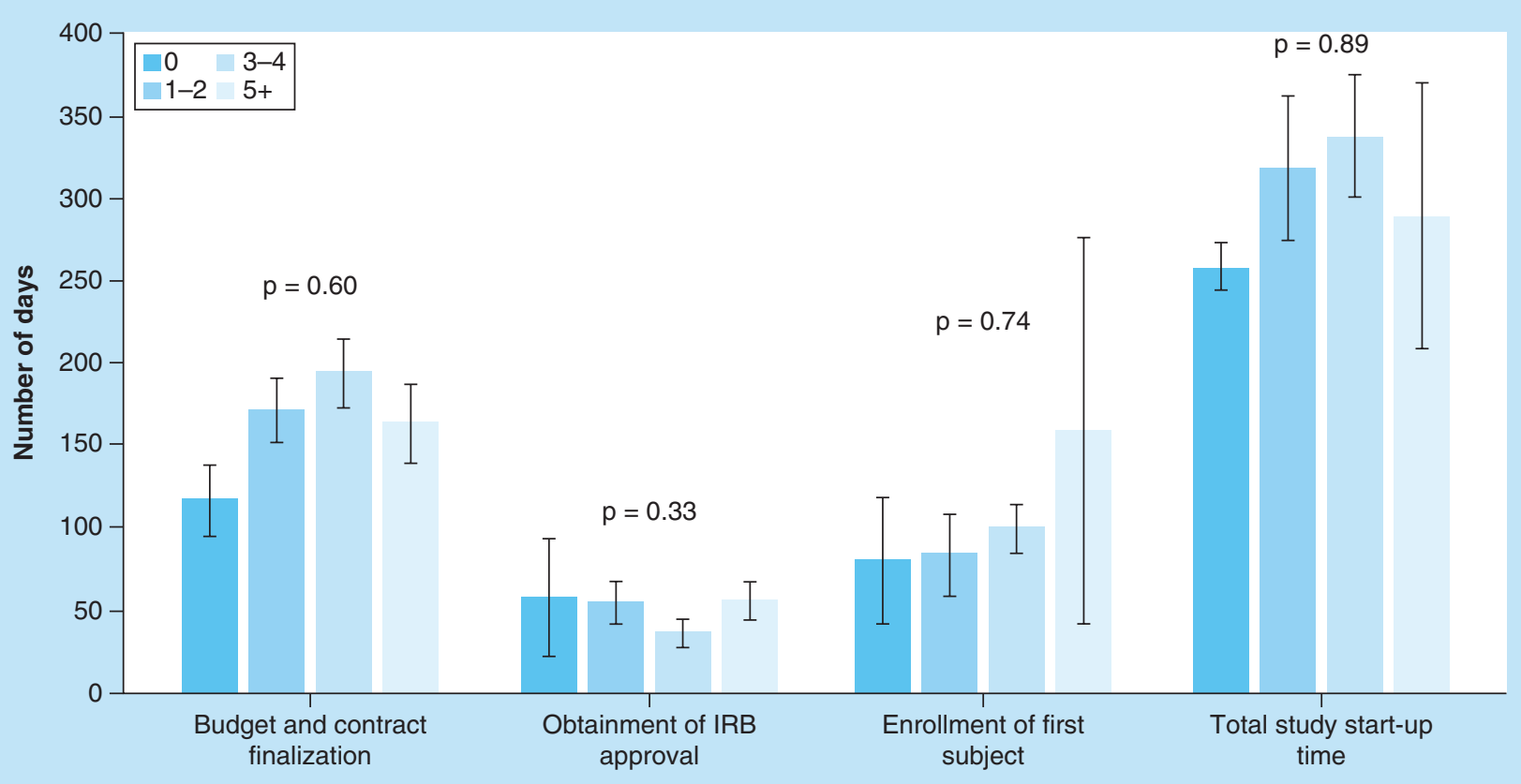

Figure 4. Comparison of number of days to finalize budget and contract, days to Institutional Review Board approval, days to first subject enrollment and total study start-up time based on number of ancillary services required by the study protocol. Total study start-up is defined as date of site receipt of the feasibility questionnaire to first subject enrollment. Bars represent standard error for each mean value. IRB: Institutional Review Board.

with those that did (194.6 vs 166.8 days, $\mathrm{p}=0.35$ ), although neither of these results was statistically significant. These results can be seen in Figure 8. Similarly, a comparison of the number of days to obtain IRB approval was compared between studies that eventually enrolled a patient and those that did not, and the results are shown in Figure 9. Studies that did enroll at least one patient took less time to obtain IRB approval on average ( 43.4 days vs $54.4, \mathrm{p}=0.40$ ). The difference was not statistically significant.

The cause-effect analysis of reasons for delay in study start-up can be seen in Figure 10, and includes factors

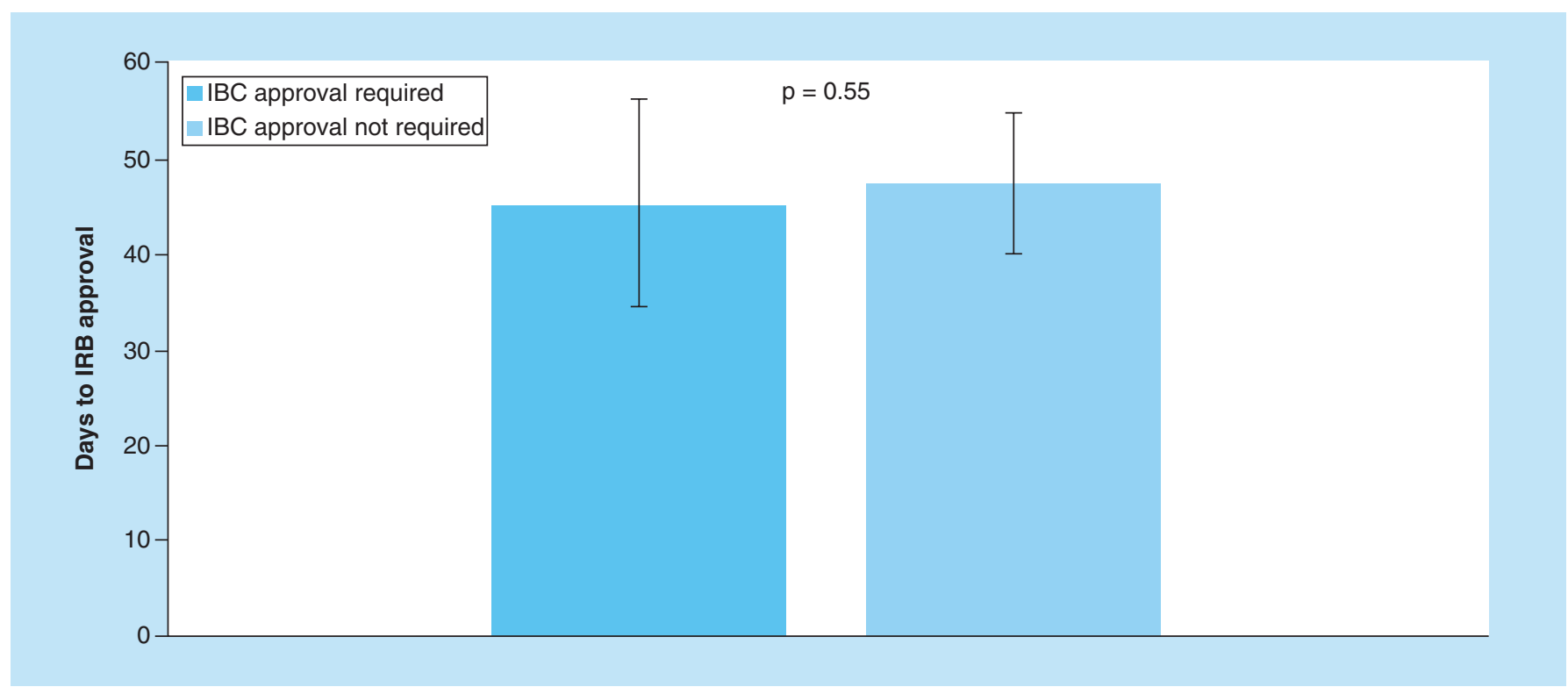

Figure 5. Comparison of number of days to Institutional Review Board approval with an Institutional Biosafety Committee approval requirement and without. Bars represent standard error for each mean value.

IBC: Institutional Biosafety Committee; IRB: Institutional Review Board. 


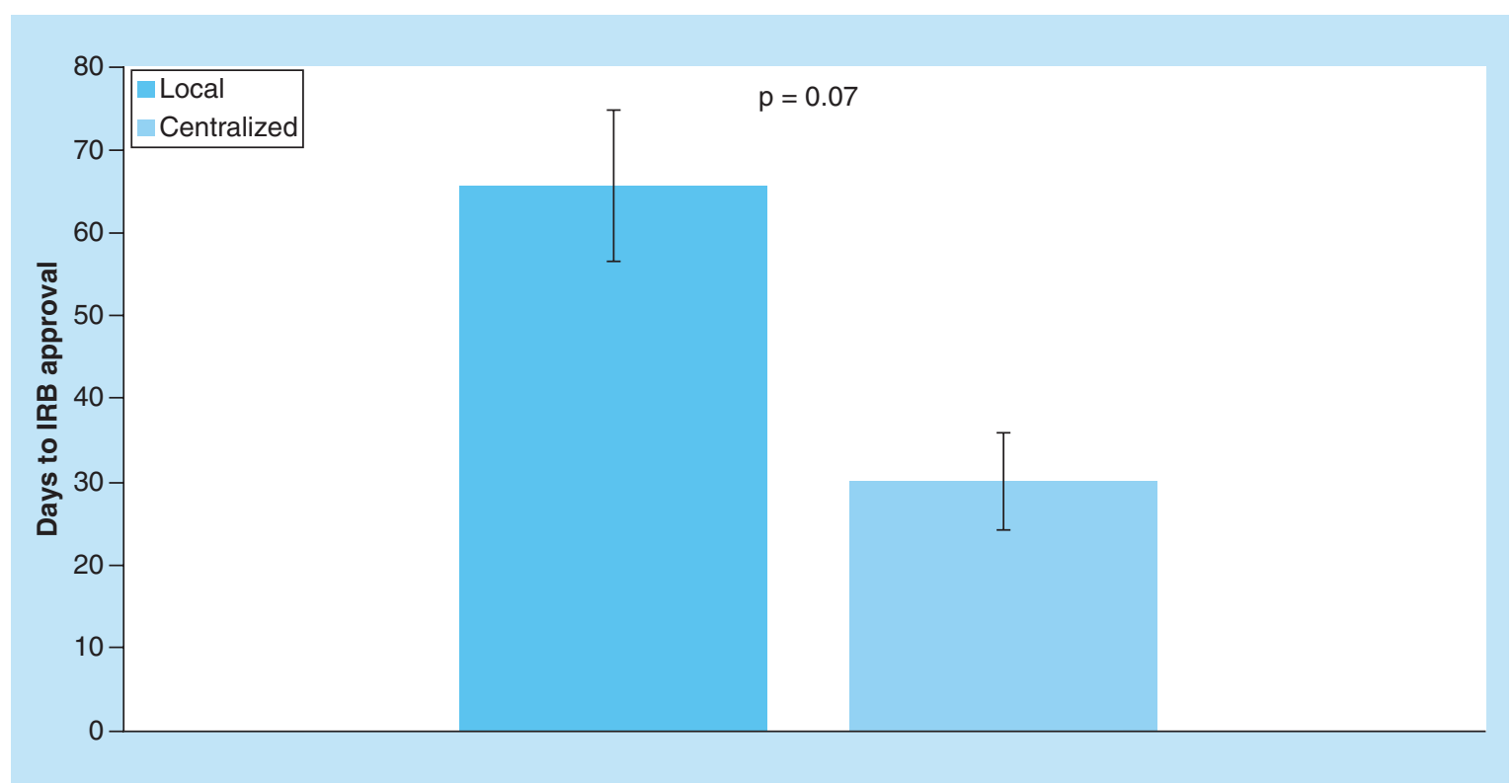

Figure 6. Comparison of number of days to Institutional Review Board approval using the local Institutional Review Board as compared to using a centralized Institutional Review Board. Bars represent standard error for each mean value.

IRB: Institutional Review Board.

related to delayed regulatory approvals, administrative issues, complications with study logistics, problems with the investigational product or pharmacy services, research personnel and study population access. Specifically, we noted common reasons for delay in start-up included difficulties in agreeing on terms of the ICF between the sponsor and site, communication with the sponsor via a CRO, numerous ancillary service or third party vendor involvement, delay in manufacturing the investigational product and a rare disease indication. In

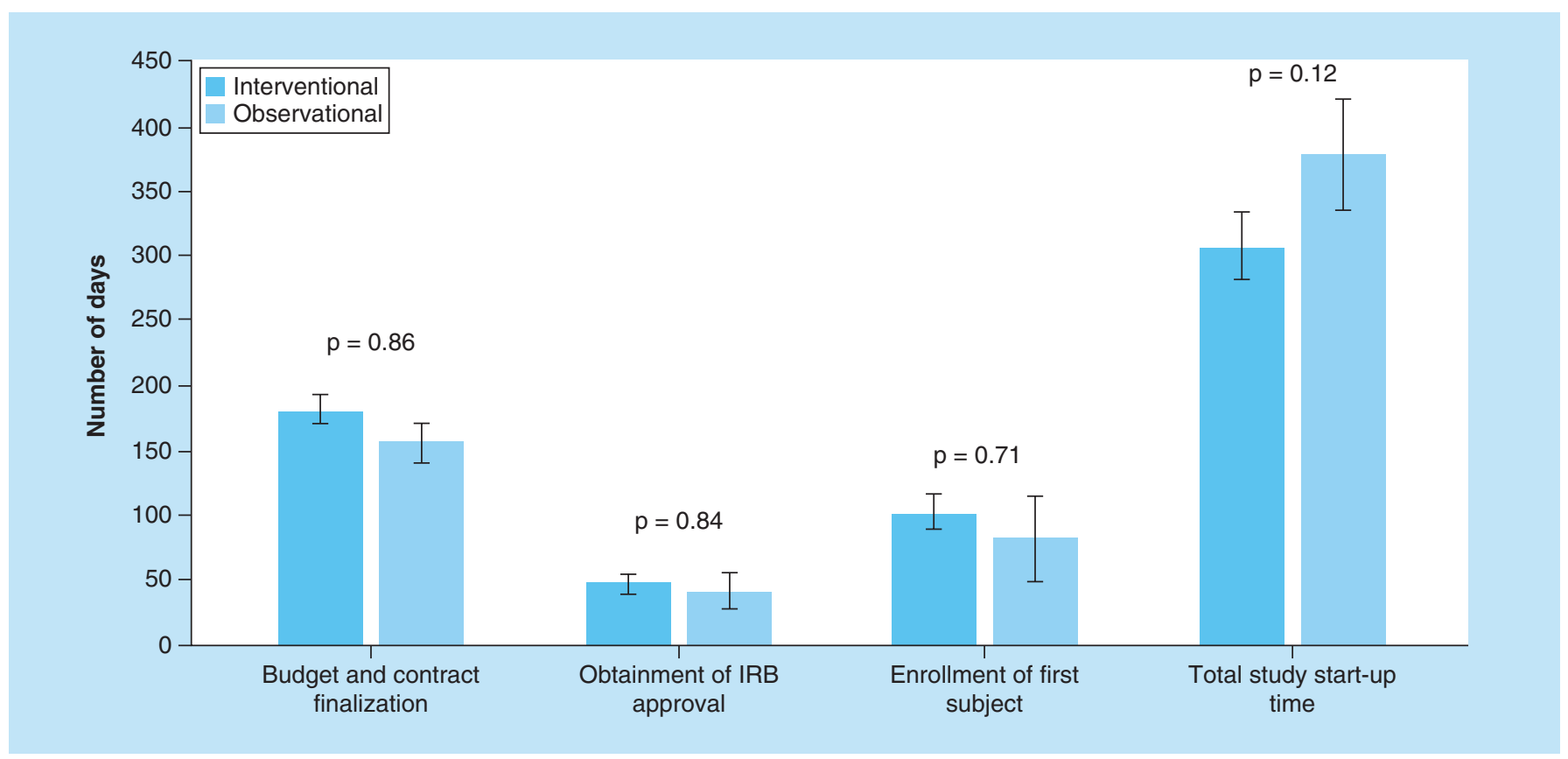

Figure 7. Comparison of number of days to finalize budget and contract, days to Institutional Review Board approval, days to first subject enrollment and total study start-up time for interventional (drug, device or biologic) studies as compared with observational studies. Total study start-up is defined as date of site receipt of the feasibility questionnaire to first subject enrollment. Bars represent standard error for each mean value. IRB: Institutional Review Board. 


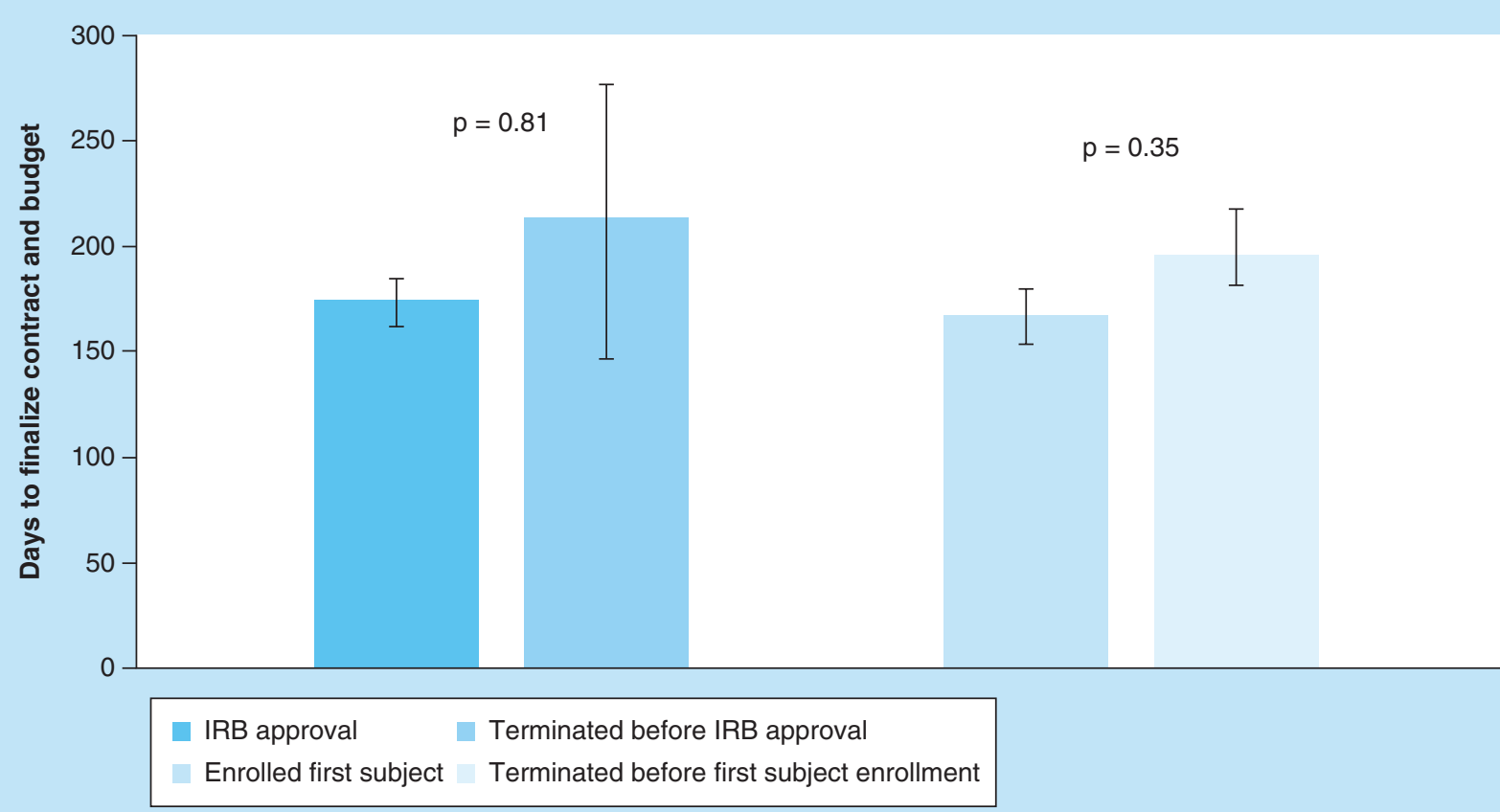

Figure 8. Comparison of the number of days taken to finalize the contract and budget for studies which obtained Institutional Review Board approval and those that were terminated before obtaining Institutional Review Board approval. The number of days for contract and budget finalization for studies that eventually enrolled a subject are compared to those that were terminated prior to first subject enrollment. Bars represent standard error for each mean value.

IRB: Institutional Review Board.

considering the observational studies, we noted that the total time between initial site contact and first patient enrollment was extended due to the fact that the studies were extensions of interventional studies at our site, and required patients to complete the interventional arm of the study before consenting for the observational study.

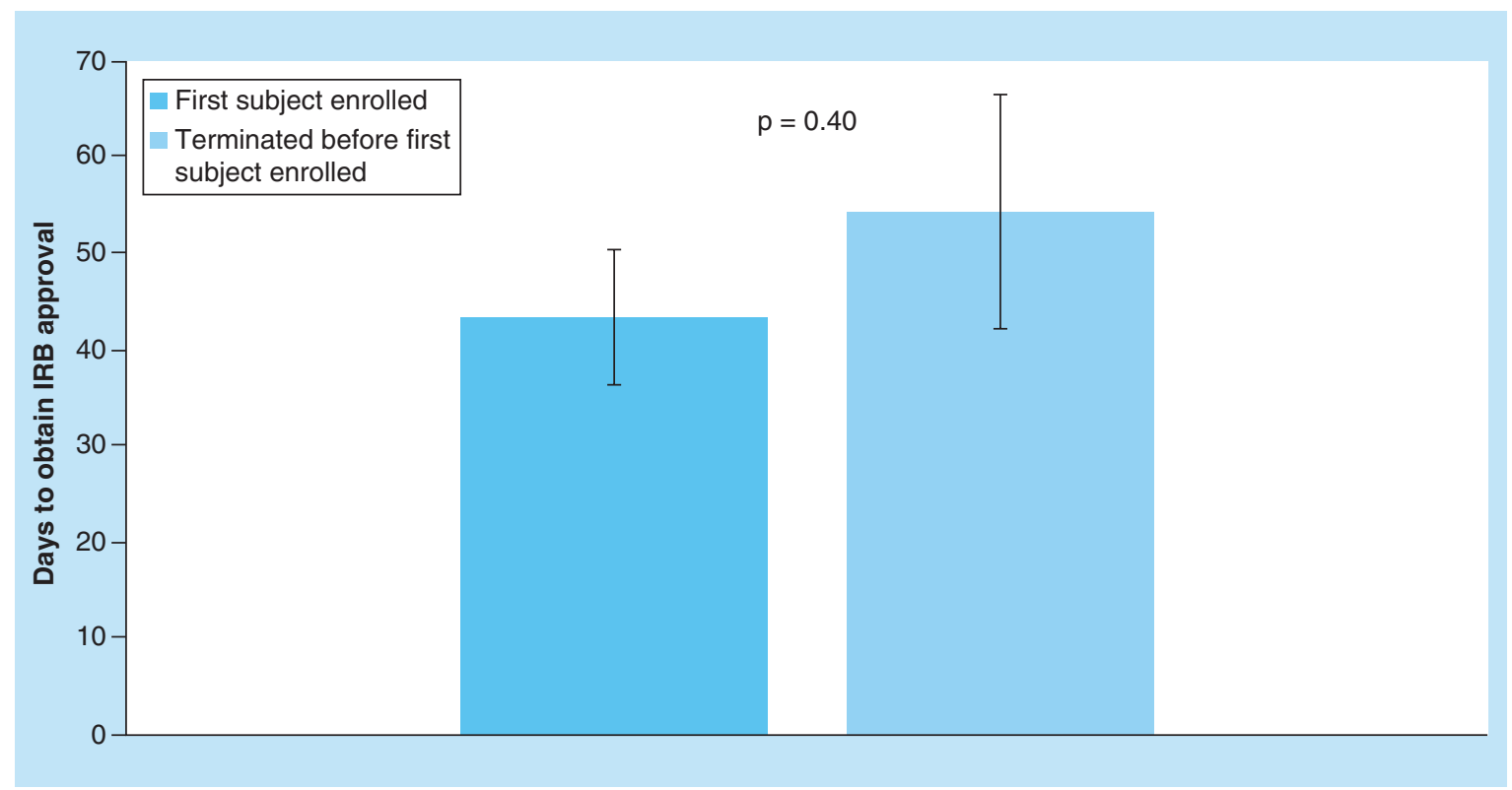

Figure 9. The number of days taken to obtain Institutional Review Board approval for studies that eventually enrolled a subject are compared with those that were terminated before the first subject was enrolled. Bars represent standard error for each mean value. IRB: Institutional Review Board. 


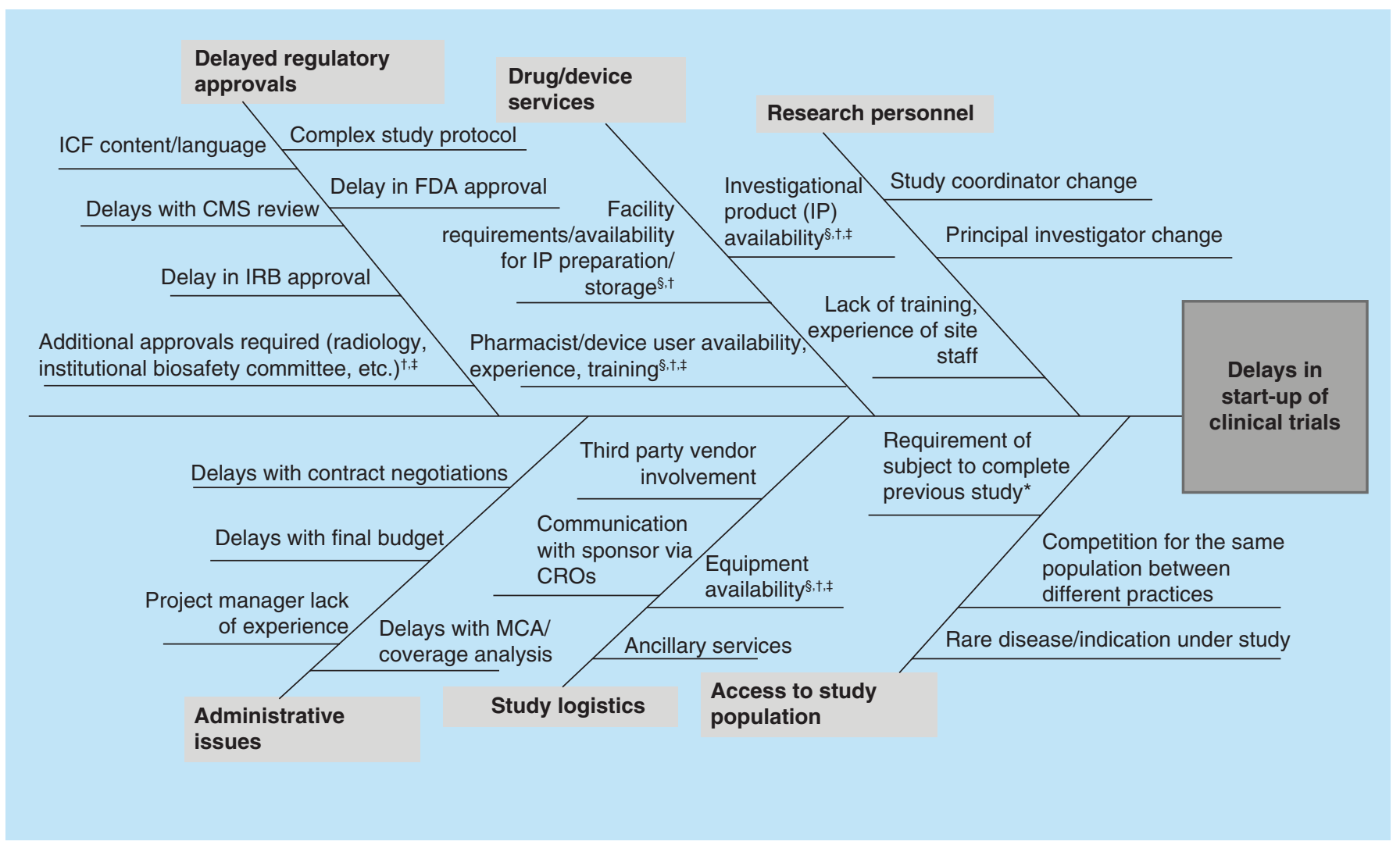

Figure 10. Cause and effect analysis of delays at the study start-up phase. Causes specific to a study type are indicated by a symbol, with the legend in the bottom right corner.

${ }^{\dagger}$ Biologic.

${ }^{\ddagger}$ Device.

${ }^{\S}$ Drug.

*Observational.

The multivariate analysis (Table 2) confirms that the use of a local IRB as opposed to a centralized IRB predicts more time to IRB approval when adjusted for use of a CRO and less project manager experience (mean ratio $[\mathrm{MR}] 2.06,95 \% \mathrm{CI} 1.56-2.56, \mathrm{p}=0.01$ ). Additionally, when considering time as a function of IRB type, time to IRB approval was significantly faster with the use of a centralized IRB as compared with a local IRB (MR 2.08, 95\% CI 1.58-2.58, $\mathrm{p}=0.01$ ). The analysis of time as a function of project manager experience, IRB type and use of a CRO is presented in Table 3.

In considering studies which were terminated during the start-up phase (i.e., did not successfully enroll a first subject), various factors played a role. Studies which did not reach a negotiated contract and budget were more likely not to have a CRO, to be a device trial, to be an interventional study, to have a project manager with less than 48 months experience and to use greater than three ancillary services (Table 4). Information about studies that did not obtain IRB approval following successful contract and budget negotiation is presented in Table 5. These terminated studies were more likely to use a CRO, be a drug or device study, be an interventional study, have a less experienced project manager, require IBC approval and use a localized IRB. Furthermore, studies that were terminated were more likely to have taken longer than both 50 and $75 \%$ of other studies of their type (drug, device, biologic or observational) to finalize the budget and contract. The features of studies that finalized a budget and contract and obtained IRB approval but did not enroll any patients are shown in Table 6. Studies that were terminated prior to patient enrollment were less likely to use a CRO, more likely to be biologic studies, were more likely to take longer than $75 \%$ of studies to finalize the contract and budget, and more likely to have taken longer than $75 \%$ of other studies of its type to obtain IRB approval.

In the Kaplan-Meier-type analysis for studies, studies which took longer than 378 days to finalize the contract and budget had a 0.89 probability of obtaining IRB approval. Similarly, studies that took longer than 308 days on the contract and budget finalization had a 0.69 probability of enrolling a first subject. The stepwise pattern based on number of days is shown in 


\begin{tabular}{|c|c|c|c|}
\hline & Mean ratio & $95 \%$ confidence interval & p-value \\
\hline \multicolumn{4}{|l|}{ Budget/contract finalization } \\
\hline Local IRB & 1.02 & $0.86-1.18$ & 0.90 \\
\hline CRO & 0.85 & $0.71-0.99$ & 0.33 \\
\hline$<48$ months project manager experience & 1.05 & $0.88-1.22$ & 0.78 \\
\hline \multicolumn{4}{|l|}{ IRB approval } \\
\hline Local IRB & 2.06 & $1.56-2.56$ & 0.01 \\
\hline CRO & 0.88 & $0.67-1.09$ & 0.62 \\
\hline$<48$ months project manager experience & 0.89 & $0.67-1.11$ & 0.65 \\
\hline \multicolumn{4}{|l|}{ First subject enrollment } \\
\hline Local IRB & 1.22 & $0.88-1.56$ & 0.50 \\
\hline CRO & 1.54 & $1.11-1.97$ & 0.15 \\
\hline$<48$ months project manager experience & 1.45 & $1.04-1.86$ & 0.24 \\
\hline
\end{tabular}

Figure 11. When considering number of days to obtain IRB approval once submitted, studies which took longer than 97 days had a 0.72 probability of ever enrolling a subject. The stepwise pattern for patient enrollment based on number of days to IRB approval is in Figure 12.

\section{Discussion}

The start-up phase of clinical trials is a resource intensive and critically important stage of research for the site, sponsor and CRO. Although the timely execution of study start-up is generally accepted as a priority by all parties, up to $86 \%$ of clinical trials experience delays in meeting the start-up timeline set by the sponsor and CRO, which results in setbacks in achieving timeline goals for the rest of the study [3]. Given the fact that maintaining a site can cost sponsors up to $\$ 2500$ per month, identifying areas of delay within the start-up process is critical in reducing the costs of clinical trials [4].

Table 3. Time to budget and contract finalization, Institutional Review Board approval, and first subject enrollment as a function of using a local Institutional Review Board, project manager experience $<48$ months and using a Contract Research Organization.

\begin{tabular}{|c|c|c|c|}
\hline & Mean ratio & $95 \%$ confidence interval & p-value \\
\hline \multicolumn{4}{|l|}{ Local IRB } \\
\hline Budget/contract finalization & 1.02 & $0.86-1.18$ & 0.90 \\
\hline IRB approval & 2.07 & $1.58-2.57$ & 0.01 \\
\hline First subject enrollment & 1.16 & $0.83-1.49$ & 0.62 \\
\hline \multicolumn{4}{|c|}{ <48 months project manager experience } \\
\hline Budget/contract finalization & 1.07 & $0.90-1.24$ & 0.66 \\
\hline IRB approval & 0.95 & $0.70-1.20$ & 0.84 \\
\hline First subject enrollment & 1.17 & $0.82-1.52$ & 0.61 \\
\hline \multicolumn{4}{|l|}{ CRO } \\
\hline Budget/contract finalization & 0.84 & $0.71-0.97$ & 0.28 \\
\hline IRB approval & 0.83 & $0.62-1.04$ & 0.49 \\
\hline First subject enrollment & 1.35 & $0.97-1.73$ & 0.29 \\
\hline
\end{tabular}


Table 4. Analysis of the features of studies which did not ultimately reach a negotiated contract and budget.

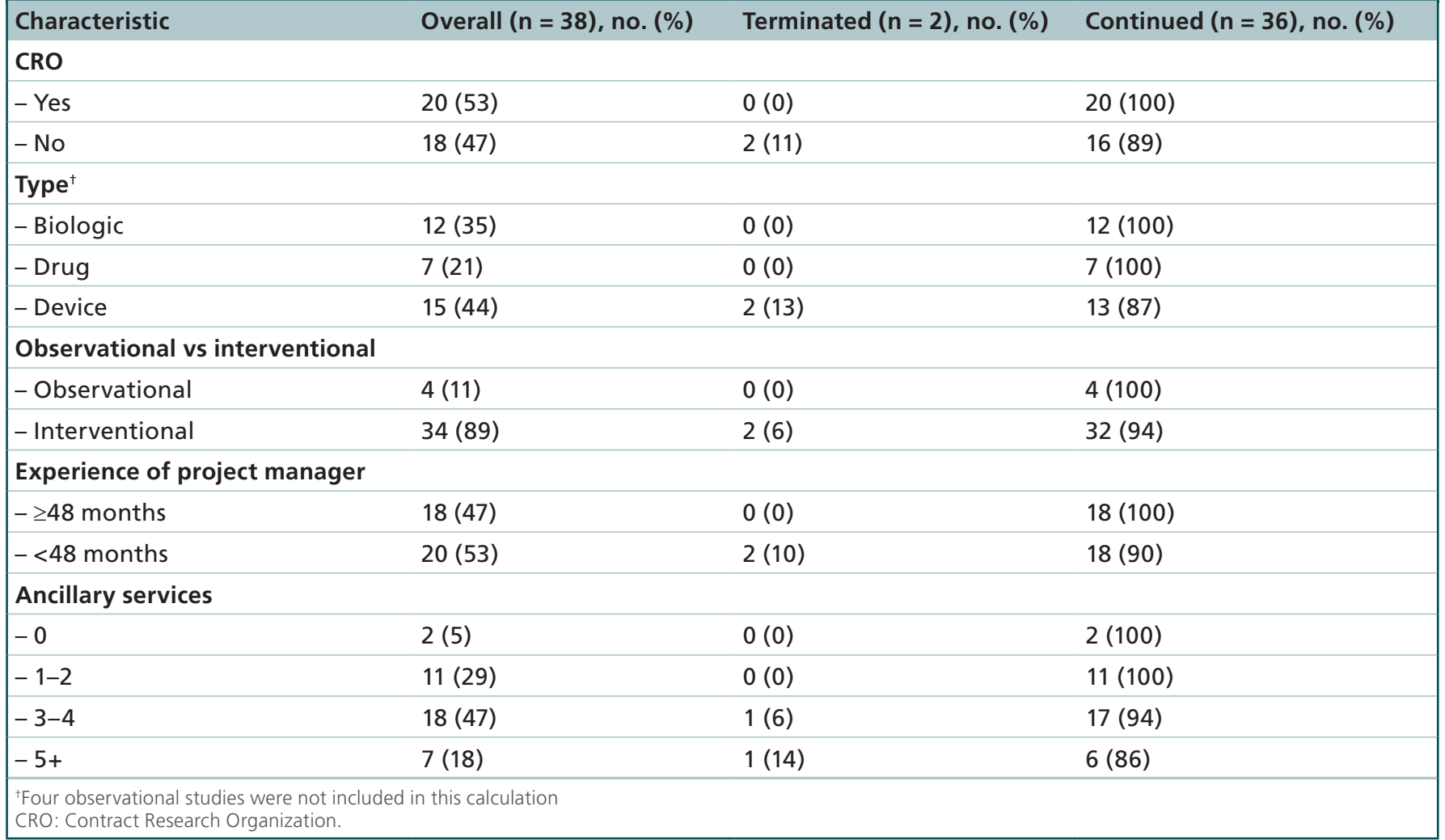

Study type did not have a consistent effect on the start-up time across all milestones, though there were some trends noted. Although device studies took the longest to finalize the budget and contract and to obtain IRB approval, they took the least amount of time to complete the total start-up cycle overall. This could be attributable to disease indication, whereby, although the administrative aspects of the start-up process are time consuming in device studies, it is less difficult to enroll patients as compared with some of the other study types. Time to enroll patients was the greatest in biologic studies, which may be reflective of the higher frequency of rarer indications associated with this study type.

Observational studies took longer overall than interventional studies, although less time to complete each of the other start-up milestones. This is attributable to the fact that two of the observational studies analyzed here were follow-up studies of an interventional trial and so despite the parallel study start-up processes, there was a delay in patient enrollment which was contingent upon completion of the interventional study.

The results of our study demonstrated that the use of CRO can both expedite and delay the start-up process, depending on which aspect is being considered. The use of a CRO decreased the amount of time for the administrative phases of the start-up, specifically the contract and budget negotiation and IRB approval. These results were confirmed by the multivariate analysis. The time to enroll the first subject and total study start-up time was longer in studies that used a CRO. This finding has been confirmed by other studies which found that the time from protocol approval to the first patient randomization was increased by $15 \%$ in studies using a CRO as compared with those managed by the sponsor internally [5]. The reasons for this are likely multifactorial. Studies that use a CRO will inevitably have more lines of communication and handoffs during the start-up phase, contributing to the overall time to enroll a subject with the introduction of additional downtime [1]. CROs are also variable, and some may be more efficient than others in accomplishing the goals of the sponsor. Additionally, studies with more complicated protocols or a more rare indication may lead sponsors to opt for using a CRO, which could inflate the number of days of the start-up cycle, at no fault of the CRO itself.

An increase in project manager experience reduced the amount of time to negotiate the contract and budget as well as the time to enroll the first patient following IRB approval, which was confirmed by the multivariate analysis. This was expected, as project managers who are knowledgeable about clinical trial execution in addition to good clinical practices and regulatory compliance mat- 
ters are more likely to be able to appropriately execute the contract and budget and communicate effectively with the institutional lawyers as well as the sponsors [2]. Furthermore, it would be expected that more experience at a single site would lead to better study selection at the site level based on a more intimate knowledge of the patient population which, in turn, could result in faster patient enrollment. The current study did show a delay in IRB approval and total study time with a more experienced project manager, which could be the result of a willingness to take on more complicated clinical trials, which requires more work during the start-up process on establishing logistics at the site. Studies with less experienced project managers were more likely to be terminated during the budget/contract and IRB approval phase, which could be the result of more difficulty with negotiations or less knowledge of how to successfully assemble an
IRB application for a particular indication, both resulting in prolonged time to approval.

The use of a centralized IRB, as compared with a local IRB at our institution, reduced the amount of time between protocol submission and approval by 35.8 days. The difference in time to approval was statistically significant in the multivariate analysis. This result is consistent with other trials, which showed an average decrease of 27 days in site approval when comparing sites using a centralized IRB to those using a local IRB, and these sites go on to enroll a first patient 21 days faster [6]. From a site standpoint, faster approval equates to earlier entry into the execution phase and more potential subject enrollment, which is a critical factor in studies with competitive enrollment. This may explain the results observed in the current data, where studies using a local IRB were more likely to be terminated before obtaining IRB

Table 5. Analysis of features of studies which did not ultimately obtain Institutional Review Board approval.

\begin{tabular}{|c|c|c|c|}
\hline Characteristic & Overall $(n=36)$, no. $(\%)$ & Terminated $(n=4)$, no. $(\%)$ & Continued $(n=33)$, no. $(\%)$ \\
\hline \multicolumn{4}{|l|}{ CRO } \\
\hline - Yes & $20(56)$ & $3(15)$ & $17(85)$ \\
\hline- No & $16(44)$ & $1(6)$ & $15(94)$ \\
\hline \multicolumn{4}{|l|}{ Type $^{+}$} \\
\hline - Biologic & $12(38)$ & $1(8)$ & $11(92)$ \\
\hline - Drug & $7(22)$ & $1(14)$ & $6(86)$ \\
\hline - Device & $13(41)$ & $2(15)$ & $11(85)$ \\
\hline \multicolumn{4}{|c|}{ Observational vs interventional } \\
\hline - Observational & $4(11)$ & $0(0)$ & $4(100)$ \\
\hline - Interventional & $32(89)$ & $4(13)$ & $28(88)$ \\
\hline \multicolumn{4}{|c|}{ Time to finalize budget/contract (75th percentile) } \\
\hline$-\geq 75 \%$ by type & $10(28)$ & $2(20)$ & $8(80)$ \\
\hline$-<75 \%$ by type & $26(72)$ & $2(8)$ & $24(92)$ \\
\hline \multicolumn{4}{|c|}{ Time to finalize budget/contract ( 50 th percentile) } \\
\hline$-\geq 50 \%$ by type & $19(53)$ & $3(16)$ & $16(84)$ \\
\hline$-<50 \%$ by type & $17(47)$ & $1(6)$ & $16(94)$ \\
\hline \multicolumn{4}{|l|}{ IBC approval required } \\
\hline - Yes & $11(31)$ & $2(18)$ & $9(82)$ \\
\hline$-\mathrm{No}$ & $25(69)$ & $2(8)$ & $23(92)$ \\
\hline \multicolumn{4}{|c|}{ Experience of project manager } \\
\hline - $\geq$ Median (48 months) & $18(50)$ & $1(6)$ & $17(94)$ \\
\hline - $<$ Median (48 months) & $18(50)$ & $3(17)$ & $15(83)$ \\
\hline \multicolumn{4}{|l|}{ Local/centralized IRB } \\
\hline - Local & $18(50)$ & $3(17)$ & $15(83)$ \\
\hline - Centralized & $18(50)$ & $1(6)$ & $17(94)$ \\
\hline
\end{tabular}


Table 6. Analysis of features of studies which did not ultimately enroll a first patient.

\begin{tabular}{|c|c|c|c|}
\hline Characteristic & Overall $(n=32)$, no. $(\%)$ & Terminated $(n=10)$, no. $(\%)$ & Continued $(n=22)$, no. (\%) \\
\hline \multicolumn{4}{|l|}{ CRO } \\
\hline - Yes & $17(53)$ & $4(24)$ & $13(76)$ \\
\hline - No & $15(47)$ & $6(40)$ & $9(60)$ \\
\hline \multicolumn{4}{|l|}{ Type $^{\dagger}$} \\
\hline - Biologic & $11(39)$ & $5(45)$ & $6(55)$ \\
\hline - Drug & $6(21)$ & $2(33)$ & $4(67)$ \\
\hline - Device & $11(39)$ & $3(27)$ & $8(73)$ \\
\hline \multicolumn{4}{|c|}{ Interventional vs observational } \\
\hline - Observational & $4(13)$ & $0(0)$ & $4(100)$ \\
\hline - Interventional & $28(88)$ & $10(36)$ & $18(64)$ \\
\hline \multicolumn{4}{|c|}{ Time to finalize budget/contract (75th percentile) } \\
\hline$-\geq 75 \%$ by type & $8(25)$ & $3(38)$ & $5(63)$ \\
\hline$-<75 \%$ by type & $24(75)$ & $7(29)$ & $17(71)$ \\
\hline \multicolumn{4}{|c|}{ Time to finalize budget/contract (50th percentile) } \\
\hline$-\geq 50 \%$ by type & $16(50)$ & $4(25)$ & $12(75)$ \\
\hline$-<50 \%$ by type & $16(50)$ & $6(38)$ & $10(63)$ \\
\hline \multicolumn{4}{|c|}{ Time to IRB approval (75th percentile) } \\
\hline$-\geq 75 \%$ by type & $8(25)$ & $4(50)$ & $4(50)$ \\
\hline$-<75 \%$ by type & $24(75)$ & $6(25)$ & $18(75)$ \\
\hline \multicolumn{4}{|c|}{ Time to IRB approval (50th percentile) } \\
\hline$-\geq 50 \%$ by type & $16(50)$ & $8(50)$ & $8(50)$ \\
\hline$-<50 \%$ by type & $16(50)$ & $2(13)$ & $14(88)$ \\
\hline
\end{tabular}

approval and studies that took longer than the median number of days to obtain IRB approval were less likely to enroll a first patient. A requirement for IBC approval did not significantly alter the time to IRB approval, suggesting that this is not a major contributor to delays in study start-up, and that the application is well-integrated to the IRB approval process at our site.

When considering studies which were terminated prior to completing the start-up process, prolonged time to complete milestones was shown to be correlative with site termination. When considering specific factors associated with early termination, many of the factors that led to delay in the start-up process increased likelihood of termination. For example, device studies were more likely to be terminated prior to contract and budget negotiation or IRB approval, however, once these were obtained, were least likely to be terminated prior to first subject enrollment as compared with the other study types. Similarly, less project manager experience is associated with more terminations in the contract/budget and IRB approval phase.
This study is not without inherent limitations. As a single center study, additional factors related specifically to the institution may play a role in start-up delay which may not be reflective of start-up at other sites. We are also limited by sample size, which limits power and statistical significance. Therefore, our intention was to present general trends, not specific guidelines. Furthermore, we did not specifically analyze factors such as clinical research associate experience level, which could have impacted administrative task completion and first subject enrollment times. Additionally, although we attempted to elucidate confounding variables using multivariate analysis, some factors may be intrinsically related and therefore skew the results. Finally, each study is different from another, and so it is difficult to identify the exact amount of time each study start-up milestone should take. Nevertheless, it is important to foster a better understanding of study start-up metrics and what factors impact the amount of time each phase will take, in order to establish more realistic timelines and have a basis of comparison among sites. 


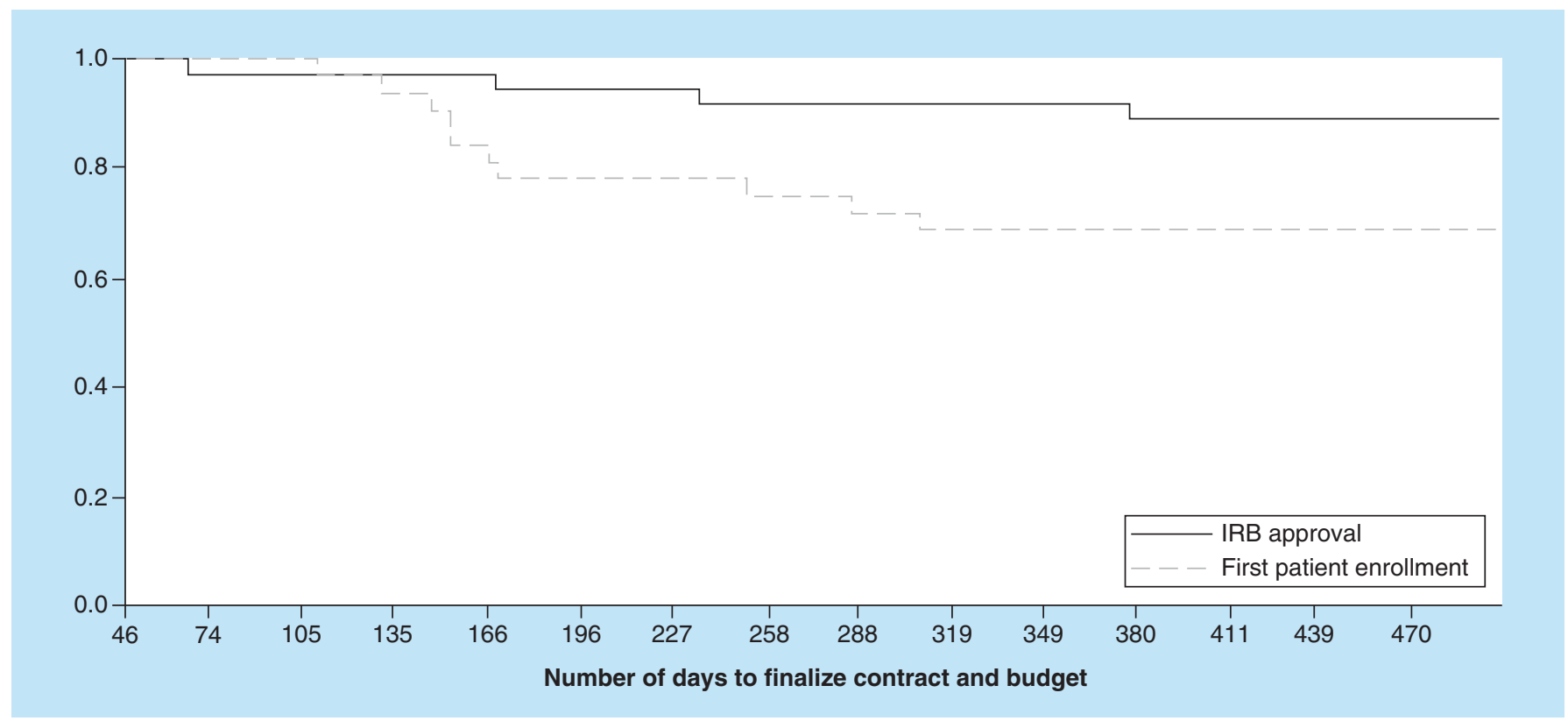

Figure 11. Kaplan-Meier-type curve for the study start-up phase based on number of days to finalize the contract and budget. The lines represent probability of ever obtaining IRB approval and probability of enrolling a first subject, respectively.

\section{Conclusion \& future perspective}

A better understanding of the study start-up phase of clinical trials at the site level can help with better adherence to trial timelines, and subsequently, reduced costs. Although some aspects of a study protocol and its execution cannot be changed, anticipation of delay with use of a local IRB, a greater number of ancillary services or a less experienced project manager, for example, can help to set more realistic guidelines for milestone achievement. Furthermore, this study provides insight as to the number of days, on average, each start-up task should take for different types of studies, as well as the probability of early site termination as a function of time and study features. Organizational structure, approval process, personnel turn around and their work load (i.e., number of clinical studies running concurrently at

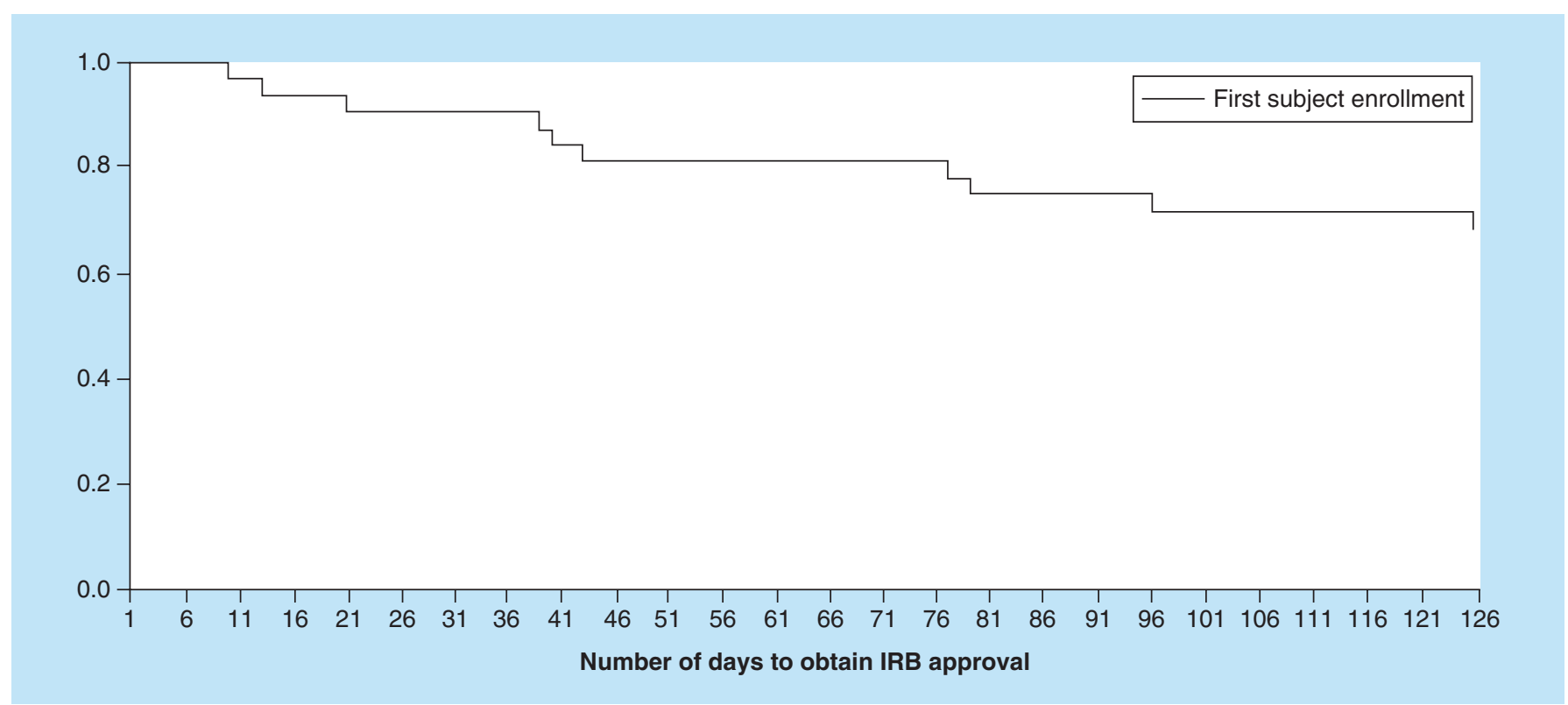

Figure 12. Kaplan-Meier-type curve for the study start-up phase based on number of days to obtain Institutional Review Board approval. The lines represent probability of eventually enrolling a first subject. IRB: Institutional Review Board. 
the site) can contribute to the delays. Certainty, multicenter studies will be beneficial to further test how different factors contribute to start-up process and ensure generalizability of results obtained in this pilot study.

\section{Financial \& competing interests disclosure}

The authors have no relevant affiliations or financial involvement with any organization or entity with a financial interest in or financial conflict with the subject matter or materials discussed in the manuscript. This includes employment, consultancies, honoraria, stock ownership or options, expert testimony, grants or patents received or pending, or royalties.

No writing assistance was utilized in the production of this manuscript.

\section{Ethical conduct of research}

The authors state that they have obtained appropriate Institutional Review Board approval or have followed the principles outlined in the Declaration of Helsinki for all human or animal experimental investigations. In addition, for investigations involving human subjects, informed consent has been obtained from the participants involved.

\section{Open access}

This work is licensed under the Creative Commons Attribution 4.0 License. To view a copy of this license, visit http://creativecommons.org/licenses/by/4.0/

\section{Authors contributions}

Krafcik BM did data collection, data analysis, literature review, manuscript writing, figures and tables creation and input into study design.

Doros $\mathrm{G}$ designed the study performed statistical analysis. Malikova MA did study design, data collection, data analysis, manuscript writing, leadership of the project for process improvement.

\section{Summary points}

\section{Aim}

- The timely execution of the start-up phase of clinical trials is crucial to efficient execution of the entire project.

- The purpose of this study was to determine factors contributing to delays and/or early termination in start-up phase to develop mitigation strategies and optimize processes.

\section{Materials \& methods}

- The start-up milestones were assessed and information about each trial was recorded for 38 studies.

- The dates were used to calculate the number of days between milestones as well as total study start-up time, which were then statistically analyzed to find differences between studies based on specific features.

\section{Results}

- Total study start-up time was the least in device trials, studies that did not use a Contract Research Organization, studies with a less experienced project manager, studies utilizing fewer ancillary services, and in interventional versus observational studies.

- The use of a centralized Institutional Review Board (IRB) versus a local IRB significantly reduced time to IRB approval.

- Studies terminated before obtaining IRB approval took longer to finalize the contract and budget than those that obtained approval.

- Similarly, studies that never enrolled a first patient took longer on average to finalize their budget and contract and obtain IRB than those that did enroll a first patient.

Conclusion \& future perspective

- Different features of individual clinical trials can lead to changes in the timeline of the study start-up process.

- A better understanding of the impact of each contributing factor allows for more realistic expectations when establishing goals and future optimization of start-up phase.

\section{References}

Papers of special note have been highlighted as:

- of interest; $\bullet \bullet$ of considerable interest

1 Schimanski C. Streamline and improve study start up. Appl. Clin. Trials 22(9), 22-25 (2013).

-• Highlights of current improvement strategies for start-up phase are provided.

2 Howley M, Malamis P. High performing study start ups. Appl. Clin. Trials (2013).

www.appliedclinicaltrialsonline.com
-. Updated metrics contributing to higher performance at start-up phase are summarized.

3 Christel M. New approaches to speeding up clinical trials what works and what doesn't. Appl. Clin. Trials (2015). www.appliedclinicaltrialsonline.com

4 Lamberti M, Brothers C, Manak D, Getz K. Benchmarking the study initiation process. Ther. Innov. Regul. Sci. 47(1), 101-109 (2013).

- Methodology of benchmarking of the study initiation process is described. 
5 Study Start-Up Takes Longer for CROs: Results from KMR group outsourcing performance study (2013). www.biospace.com/News
6 transformation initiative (2012).
www.ctti-clinicaltrials.org/files 